\title{
Article \\ A Single Assistive Profile Applied by a Passive Hip Flexion Device Can Reduce the Energy Cost of Walking in Older Adults
}

\author{
Fausto Antonio Panizzolo ${ }^{1, *}$, Eugenio Annese ${ }^{1}$, Antonio Paoli ${ }^{2}$ (D) and Giuseppe Marcolin ${ }^{2}$ (D) \\ 1 Moveo srl, Viale dell'Industria 19, 35129 Padova, Italy; eugenio.annese@gmail.com \\ 2 Department of Biomedical Sciences, University of Padova, Via Marzolo 3, 35131 Padua, Italy; \\ antonio.paoli@unipd.it (A.P.); giuseppe.marcolin@unipd.it (G.M.) \\ * Correspondence: fausto@moveowalks.com
}

Citation: Panizzolo, F.A.; Annese, E.; Paoli, A.; Marcolin, G. A Single Assistive Profile Applied by a Passive Hip Flexion Device Can Reduce the Energy Cost of Walking in Older Adults. Appl. Sci. 2021, 11, 2851. https://doi.org/10.3390/app11062851

Academic Editor: Giuseppe Andreoni

Received: 18 February 2021

Accepted: 19 March 2021

Published: 23 March 2021

Publisher's Note: MDPI stays neutral with regard to jurisdictional claims in published maps and institutional affiliations.

Copyright: (c) 2021 by the authors. Licensee MDPI, Basel, Switzerland. This article is an open access article distributed under the terms and conditions of the Creative Commons Attribution (CC BY) license (https:// creativecommons.org/licenses/by/ $4.0 /)$.

\begin{abstract}
Difficulty walking in older adults affects their independence and ability to execute daily tasks in an autonomous way, which can result in a negative effect to their health status and risk of morbidity. Very often, reduced walking speed in older adults is caused by an elevated metabolic energy cost. Passive exoskeletons have been shown to offer a promising solution for lowering the energy cost of walking, and their simplicity could favor their use in real world settings. The goal of this study was to assess if a constant and consistent low torque applied by means of a passive exoskeleton to the hip flexors during walking could provide higher and more consistent metabolic cost reduction than previously achieved. Eight older adults walked on a treadmill at a constant speed of $1.1 \mathrm{~m} / \mathrm{s}$ with and without the hip assistive device. Metabolic power and spatiotemporal parameters were measured during walking in these two conditions of testing. The hip assistive device was able to apply a low torque which initiates its assistive effect at mid-stance. This reduced the metabolic cost of walking across all the participants with respect to free walking $(-4.2 \pm 1.9 \%$; $p=0.002)$. There were no differences in the spatiotemporal parameters reported. This study strengthened the evidence that passive assistive devices can be a valuable tool to reduce metabolic cost of walking in older adults. These findings highlighted the importance of investigating torque profiles to improve the performance provided by a hip assistive device. The simplicity and usability of a system of this kind can make it a suitable candidate for improving older adults' independence.
\end{abstract}

Keywords: exoskeleton; hip assistance; elderly population; metabolic cost; human-robot interface; gait analysis

\section{Introduction}

The reduction in walking speed experienced by older adults influences quality of life, health status, and predicts life expectancy [1,2]. Very often, both biomechanics and movement control are altered leading to changes of specific walking movement parameters. Trunk exhibits a more flexed posture, there is a decreased hip extension and ankle plantarflexion, and the disturbance of movement control causes a larger stride length variability and timing issues, such as a loss of the rhythm and difficulty transitioning from stance to swing [3]. This difficulty walking in older adults affects their independence and ability to execute daily tasks in an autonomous way [3].

One of the leading explanatory factors proposed for the reduced walking speed in older adults is an elevated metabolic energy cost of walking [4]. Indeed, elevated walking energy costs have been shown to result in a general avoidance of walking and other activities in the elderly [4]. It has been suggested that when the energy required for a specific task comprises a larger fraction of the energy available to the body, older adults experience these tasks as more intense and more fatigue inducing [5,6]. Lowering walking energy cost and increasing preferred walking speed can significantly improve health, quality of life, and life expectancy in older adults [7]. 
In the last years exoskeletons have been developed with the purpose of reducing metabolic cost during walking [8-11]. To achieve this goal, engineers developed different devices which applied an assistive torque to the lower limbs: some of them are active, powered by batteries or external actuators $[9,10,12]$, while others are passive [11,13-16], storing and releasing part of the mechanical energy generated by the human body at specific phases of the gait cycle by means of elastic mechanisms.

Despite research conducted in this field, only 13 studies to date have reported successful reduction of metabolic cost of walking while using an exoskeleton, as listed in a recent detailed review on the exoskeletons' expansion [17]. Some of these studies have also shown that individuals exhibit significant physiological and neurological differences which can lead to a large variety, and sometime contrasting metabolic responses when using the same assisted device $[9,11,18]$. To this extent, Collins and colleagues [11] reported a large variability of metabolic cost reductions between the participants involved in their study. These results were obtained from people walking with a passive ankle device varying the level of stiffnesses applied to the joint by means of a spring acting in parallel with the Achilles tendon [11]. Another study involving a powered hip exoskeleton [18] highlighted how time spent walking with the exoskeleton affects metabolic cost reduction, and how this familiarization time changes on an individual basis. Lastly, Mooney et al. [9] reported a reduction of $8 \pm 3 \%$ while walking with an active ankle exoskeleton relative to not wearing the exoskeleton.

Previous literature [17] has underlined the importance of control architectures that are able to provide subject-specific devices' level of assistance to optimize metabolic savings for each individual $[19,20]$. To this extent, Zhang and colleagues [19] conducted real time optimization of the torque patterns using an ankle exoskeleton emulator system which tuned four parameters: peak torque, timing of peak torque, and rise and fall timing. Ding and colleagues [20] used Bayesian optimization to adapt peak and fall timing of hip extension assistance using a soft exosuit. The real time optimizations involved in these studies substantially improved the metabolic saving during walking with an average reduction of $\sim 20 \%$. Although of relevant scientific value, the findings of the aforementioned studies were still far from being translated in a real-world setting, mainly due to the presence of a tethered system powered by an external unit, needed to drive the joints' optimization assistance.

Passive exoskeletons could have a larger application in daily tasks as they do not present the same shortcomings of active systems $[11,21]$. They have the crucial advantage of a smaller encumbrance and lighter weight. They are also more affordable and do not require trained personnel to employ the device. On the other hand, they still face the limitation of a reduced control architecture with respect to active systems. The lack of a control architecture aiming at optimizing users' external assistance for a passive exoskeleton shows the need to find a suitable torque profile across participants in order to provide consistent metabolic cost reductions.

As such, the present study examined the effect of walking with a passive exoskeleton that assists hip flexion on the metabolic cost in older adults. The aim of this study was to investigate the effect of a specific assistive torque profile applied at the hip, which initiates its supporting action at a later percentage of the gait cycle, with respect to previously investigated assistive profiles [15]. It was hypothesized that this assistive profile would provide further and more consistent metabolic reduction across participants than previously achieved, in accordance with simulation studies [22] suggesting a profile of this kind for assisting hip flexion.

\section{Materials and Methods}

\subsection{Participants}

Eight male older adults (age: $63.5 \pm 5.9 \mathrm{yr}$; height: $1.70 \pm 0.04 \mathrm{~m}$; mass: $74.0 \pm$ $8.1 \mathrm{~kg}$, mean $\pm \mathrm{SD}$ ) volunteered for this study. Only participants free from back pain, cardiovascular, respiratory, and acute lower limb musculoskeletal diseases were considered 
eligible for the study. Informed consent was obtained from all participants involved in the study and they were free to withdraw from the study at any time. The study was conducted according to the guidelines of the Declaration of Helsinki, and approved by the Ethical Committee of the Department of Biomedical Sciences of the University of Padova (HEC-DSB/07-18).

\subsection{Experimental Design}

Each participant completed four walking bouts of 5-min each: two wearing the Exoband (EXO) and two without (FreeWalk). The walking sessions were performed on a treadmill (RUN 1000 EXCITE MED, Technogym, Cesena, Italy) at a fixed speed of $1.1 \mathrm{~m} / \mathrm{s}$ as this speed is within the range of preferred walking speeds reported by older adults [23,24]. A 4-min standing trial was performed before any walking condition to collect baseline metabolic power. After a short warm-up, participants underwent four 5-min bouts of walking. FreeWalk condition was performed at the beginning and at the end of the walking protocol. Following the first FreeWalk trial, the two EXO trials were performed. Between each bout of walking trials with Exoband a researcher checked if any loss in preloading occurred. If it was the case, the preload was corrected. After each walking condition, participants had the opportunity to take an adequate period of rest to avoid earlier onset of fatigue.

\subsection{Exoband Design}

The Exoband was first presented in [15]. The model used in this study (Figure 1) has minimal differences from the one presented in the previous work, as such a short description of it is presented below. The Exoband is mainly composed of textile and includes also 3D printed parts. Its design makes the device lightweight (630 $\mathrm{g}$ for the medium size) and easy to wear. It is made of three main components: a waist belt and two thigh parts. An elastic element connects each thigh part to the waist belt. A ratchet strap placed in series with the elastic element allows the changes in length of the elastic element. Researchers varied the amount of force exerted to the leg, adjusting the length of the ratchet strap, as described below.
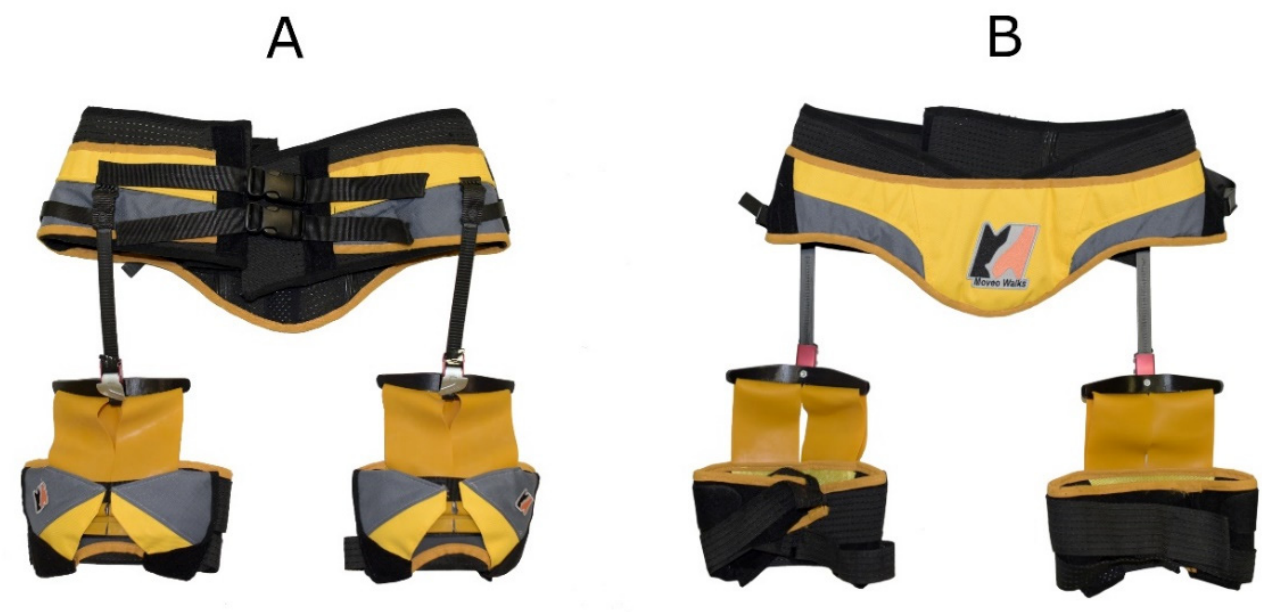

Figure 1. Front (A) and rear (B) view of Exoband, the passive device employed in the study.

\subsection{Exoband Force Setup}

The following procedure was used to set the force applied by the Exoband before the EXO condition. Participants were asked to keep an upright standing posture while the length of the elastic element was tensioned in order to obtain a constant reading of force $(9.8 \pm 1.1 \mathrm{~N})$ on the customized software used to collect force data (Labview, National Instrument, Austin, TX, USA). 


\subsection{Data Collection and Analysis}

The force exerted by the Exoband was measured by means of a load cell (ISB SBeam Force Sensor, Flintec, Hudson, MA, USA), inserted between the belt and the elastic components (Figure 2). Spatio-temporal parameters were recorded using the OptoGait system (Microgate, Italy) placing its two optical bars on each side of the treadmill. Load cells and optical measurement system were synchronized by means of a trigger box sending a $5 \mathrm{~V}$ square pulse. An overview of the experimental setup is shown in Figure 2. Only the last two-minutes of each five-minute bout were recorded and then used for the analysis. The average between the last two-minutes of the two bouts of each condition was considered for analysis and is reported in the results section. Force data relative to the right leg were segmented and normalised to $0-100 \%$ of the gait cycle using the heel-strike event detected by the OptoGait system. To obtain an estimate of the torque applied by the Exoband to the users the moment arm was measured on five participants. The obtained average value $(0.08 \pm 0.01 \mathrm{~m})$ was then multiplied by force data collected by the load cells. All the above analysis was conducted by means of a customized software (MATLAB, The MathWorks Inc., Natick, MA, USA). Metabolic cost was assessed by indirect calorimetry by means of a gas analysis machine (Vmax, SensorMedics, US). Carbon dioxide and oxygen rate were averaged across the last two minutes (3-5 $\mathrm{min}$ ) of each walking condition and then used to calculate metabolic power using the Brockway equation [25]. Net metabolic power of the two walking conditions was obtained by subtracting the standing metabolic power from the walking metabolic power of each condition and then normalising it by the body mass of each participant. FreeWalk and EXO conditions presented in the results were obtained as an average of the respective two bouts of walking.

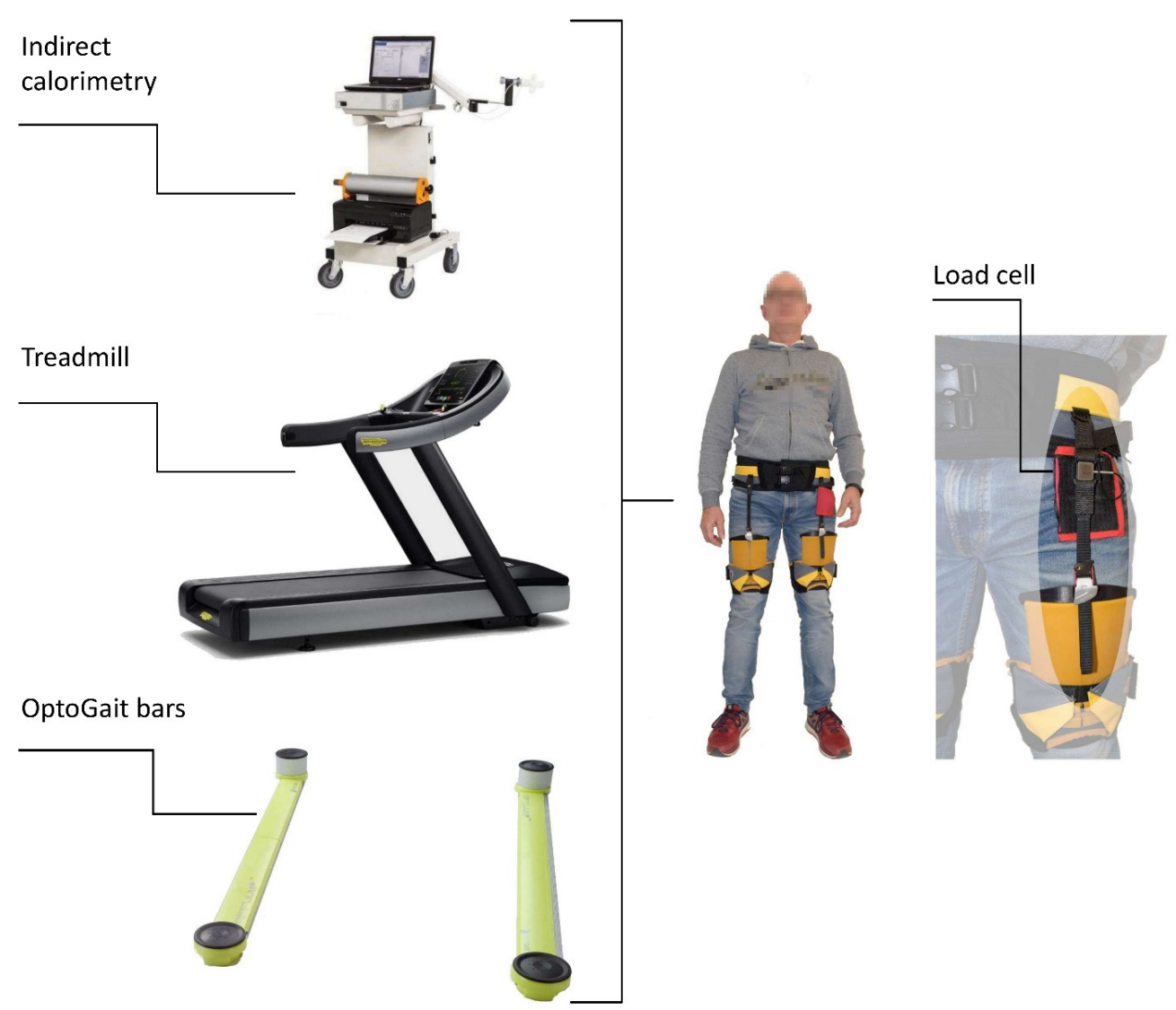

Figure 2. Overview of the experimental instrumentation adopted in the study. 


\subsection{Statistical Analysis}

Statistical analysis was conducted in GraphPad Prism version 4.00 for Windows (GraphPad Software, San Diego, CA, USA). All the collected data passed the KolmogorovSmirnov normality distribution test, and therefore a two-tailed paired Student's $t$-test was used to assess difference in metabolic power between the FreeWalk and the EXO condition. Moreover, $t$-test was used also to assess differences between spatiotemporal parameters (stance phase, swing phase, step length and stride length). The significance level was set at $p<0.05$ for all the analyses. Cohen's $d$ was calculated with $G^{*}$ Power 3.1.9.2 software [26] and evaluated as trivial $(\mathrm{d} \geq 0.19)$, small $(0.2 \leq \mathrm{d} \leq 0.49)$, medium $(0.50 \leq \mathrm{d} \leq 0.79)$ and large $(\mathrm{d} \geq 0.80)[27]$.

\section{Results}

\subsection{Metabolic Cost and Spatio-Temporal Parameters}

Metabolic power during standing had an average value of $1.3 \pm 0.3 \mathrm{~W} / \mathrm{kg}$. Net metabolic power in the EXO condition was significantly lower than in the FreeWalk $(2.99 \pm 0.44 \mathrm{~W} / \mathrm{kg}$ vs. $3.11 \pm 0.49 \mathrm{~W} / \mathrm{kg}, p=0.002$, effect size $=0.26)$, this resulted in an average reduction of $-4.2 \pm 1.9 \%$ with respect to free walking (Figure 3 ). Each participant's net metabolic power for both testing conditions is presented in Figure 4. Walking with the Exoband did not alter the spatio-temporal parameters assessed (stance and swing phase, step, and stride length) with respect to FreeWalk condition (Table 1).

A

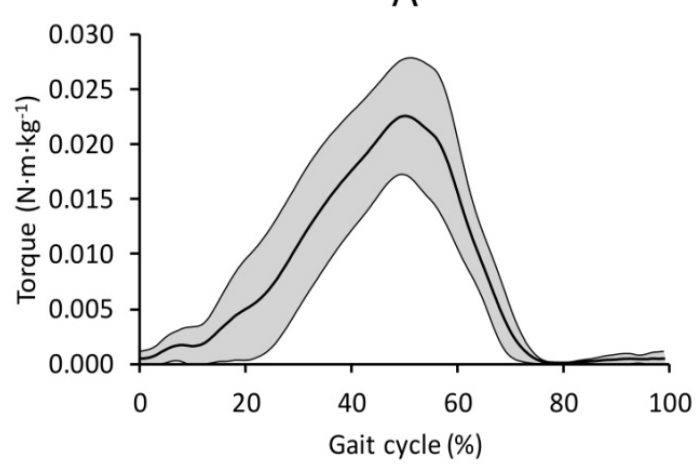

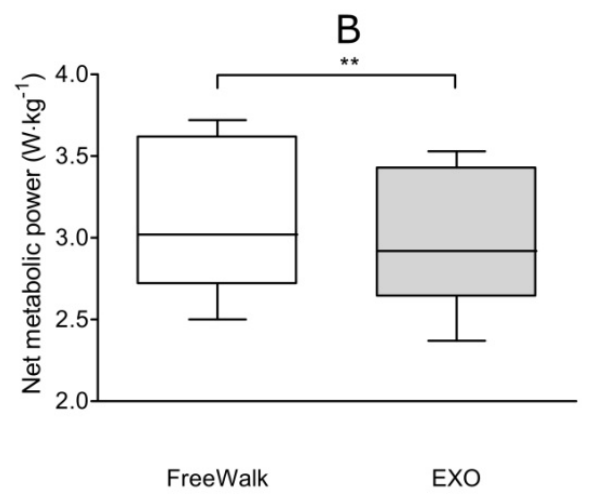

Figure 3. Average torque profile applied by Exoband in the gait cycle (A). Net metabolic power in the FreeWalk condition (white) and in the EXO condition (grey) (B). ${ }^{* *}$ indicates a significant reduction $(p<0.01)$ of the metabolic power using Exoband.

Table 1. Spatio-temporal parameters for the two conditions of testing. Data are presented as means $\pm \mathrm{SD}$.

\begin{tabular}{ccc}
\hline & FreeWalk & EXO \\
\hline Stance phase [\%] & $62.8 \pm 1.9$ & $62.7 \pm 1.8$ \\
Swing phase [\%] & $37.2 \pm 1.9$ & $37.3 \pm 1.8$ \\
Step length [cm] & $72.1 \pm 4.3$ & $71.9 \pm 4.2$ \\
Stride length [cm] & $144.2 \pm 8.6$ & $143.8 \pm 8.4$ \\
\hline
\end{tabular}




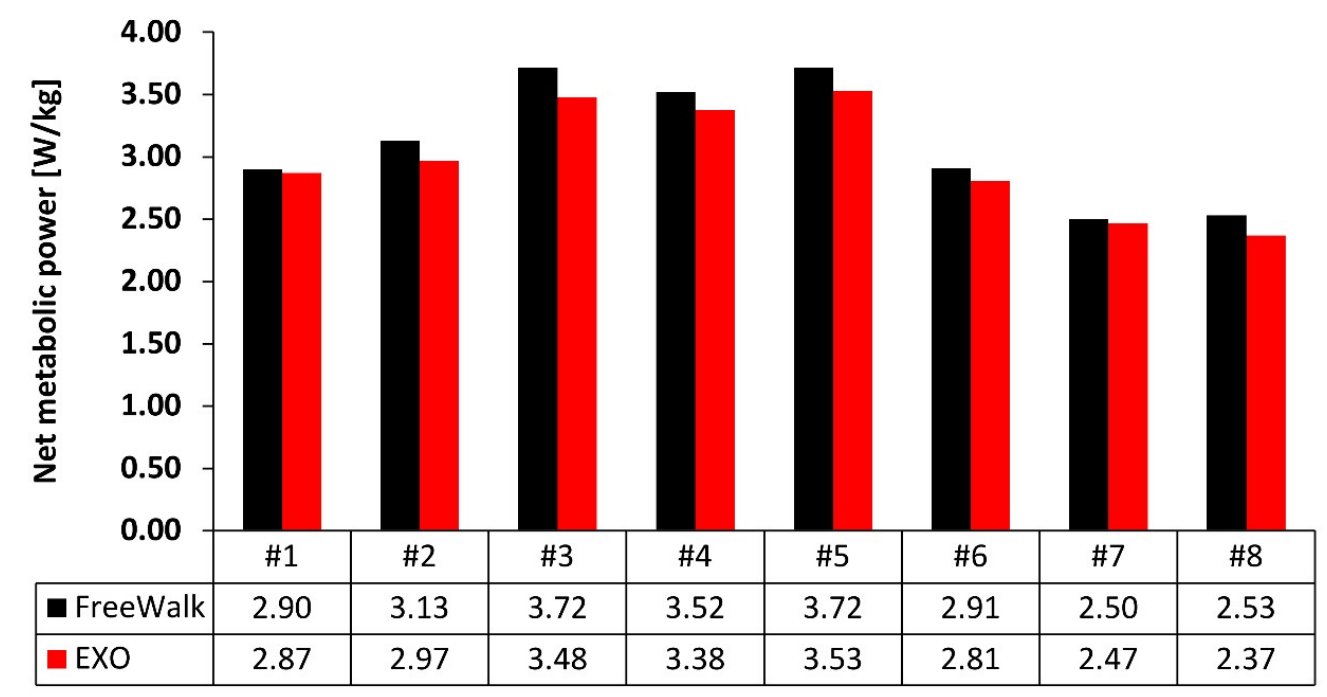

Figure 4. Net metabolic power for each participant during the two conditions of testing. FreeWalk condition (black) and EXO condition (red).

\subsection{Torque Assistance}

Peak torques were consistent during the walking trials. The average torque profiles for both the right and left leg obtained during the EXO condition are displayed in Figure 3. Onset of torque profile was at $21.1 \pm 5.0 \%$, and peak torque was at $51.6 \pm 3.2 \%$ of the gait cycle.

\section{Discussion}

The aim of this study was to investigate the effect on metabolic cost of a specific torque profile assisting hip flexion during walking. Results indicated that walking with Exoband significantly reduced the metabolic cost with respect to free walking. Further, the specific hypothesis that the investigated assistive profile would have provided more consistent metabolic reduction across participants than previously achieved [15] was verified. In the present work, each participant reported a reduced metabolic cost while walking with Exoband, while in a previous work involving a passive system [15] not all the participants were able to benefit from the use of the device thus reporting a reduced metabolic cost. The hypothesis was also confirmed by a slightly higher reduction in metabolic cost $(4.2 \%$ vs. $3.3 \%)$ and a lower SD (1.9\% vs. $3.0 \%)$. The variability of metabolic cost responses displayed by users walking with exoskeletons has been reported by several other studies, thus motivating the need to develop optimization strategies to improve users' metabolic outcomes [17]. The interesting finding of this work relies on the fact that a unique and constant assistive profile was able to provide a consistent reduction across all participants. This can have practical advantages in tuning the assistance of these devices thus favoring the adoption of simpler passive devices, which are lacking of any control architecture. Furthermore, these results are of particular interest considering that a lower peak torque was applied in this work than before $(0.023 \mathrm{Nm} / \mathrm{kg}$ vs. $0.042 \mathrm{Nm} / \mathrm{kg})$, thus highlighting the key role of a later onset timing ( $20 \%$ vs. $\sim 6 \%)$ in the reduction of metabolic cost provided using Exoband.

Few studies have been conducted on the metabolic cost of walking involving passive assistive devices, the outcomes and designs adopted are summarized in Table 2. Although the results in term of metabolic cost are in line with previous work on passive exoskeletons $[11,15,28,29]$, it has to be highlighted that all the previous studies applied a higher assistive torque than the present study. Moreover, some of the work investigated the effect of springs of different stiffness assisting the hip joint $[28,29]$ but considering the range evaluated, peak torques applied were higher than the present study. This seems to fur- 
ther suggest the importance of exploring different onset timing other than the magnitude of assistance.

Table 2. State of the art of passive assistive devices. The table includes information on the joint assisted, peak torque and mass of device.

\begin{tabular}{|c|c|c|c|c|}
\hline Study & Joint Assisted & $\begin{array}{l}\text { Peak Torque } \\
{[\mathrm{Nm} / \mathrm{kg}]}\end{array}$ & Mass [kg] & $\begin{array}{l}\text { Net Metabolic } \\
\text { Cost [\%] }\end{array}$ \\
\hline $\begin{array}{l}\text { Collins } \\
\text { et al. } 2015 \text { [11] }\end{array}$ & Hip & $\sim 0.25$ & 0.91 & -7.2 \\
\hline $\begin{array}{l}\text { Panizzolo } \\
\text { et al. } 2019 \text { [15] }\end{array}$ & Hip & $\sim 0.04$ & 0.65 & -3.3 \\
\hline $\begin{array}{c}\text { Xiong } \\
\text { et al. } 2019 \text { [29] }\end{array}$ & Hip and ankle & $\mathrm{N} / \mathrm{A}$ & 1.95 & -7.6 \\
\hline $\begin{array}{c}\text { Etenzi } \\
\text { et al. } 2020 \text { [13] }\end{array}$ & Ankle and knee & $\sim 0.05$ & 2.80 & +23 \\
\hline $\begin{array}{c}\text { Barazesh } \\
\text { et al. } 2020 \text { [28] }\end{array}$ & Hip and knee & $\mathrm{N} / \mathrm{A}$ & $\sim 1.79$ & -4.7 \\
\hline Current study & Hip & $\sim 0.02$ & 0.63 & -4.2 \\
\hline
\end{tabular}

Spatiotemporal parameters were found to be unaltered between the two conditions of testing (Table 1), indicating that walking with Exoband has no effect on these variables. This confirms the finding that the passive nature of the system did not impose a different kinematics to the elderly, and this could be a sign of a preserved walking comfort in the use of this device [15], regardless of the different assistance applied in the present study. In addition, previous work has shown that when step length is different than the preferred one, metabolic cost of walking increases [30]. Unfortunately, other works involving passive hip systems [28,29] did not report spatiotemporal parameters, thus preventing a direct comparison between devices on these parameters.

This work reports interesting outcomes with respect to metabolic cost reduction associated with the use of a passive assistive device. Nevertheless, at this stage, the lack of muscle-level data prevents us to drive conclusions on the determinants of these findings. Spatiotemporal parameters were found to be unaltered between the two conditions, but this does not exclude the presence of joint-level differences reflecting altered muscle dynamics. The earlier onset of hip flexors assistance could have been responsible for the reduced metabolic cost as suggested from simulation studies on load carriers [22]. The authors of this work suggests that there might have been a decrease in the peak moment of the iliacus and the rectus femoris, which in combination with a reduced activation of the plantarflexors, could have been responsible for a reduced metabolic cost in a hip flexion assistive device.

Last, it is relevant to mention a couple of limitations of the present work. First, the lack of training session could have reduced the possible energetic benefits provided by the device. However, training effects have been shown only on young healthy subjects and with the employment of powered exoskeletons [18,31,32], and thus the effect with a passive device on older adults is yet to be demonstrated. Second, due to the small effect size obtained, future studies with a larger sample size are warranted to deepen the effect of the Exoband on reducing the energy cost of walking in older adults.

\section{Conclusions}

The present study contributes to expand the knowledge on the research of passive assistive devices, further strengthening the evidence that they can represent a viable solution to augment human walking in older adults, as reported by the reduced metabolic cost. This work also highlights the importance of investigating torque profiles, in terms of magnitude and onset of activation, to improve the performance in this kind of systems and not only in active devices which focus on improving their control architecture. In particular, results showed that the assistive torque proposed was suitable to provide 
metabolic reduction across all participants. This can have practical application in selecting an initial assistive profile with a user wearing the device for the first time. Although further studies involving more participants might be needed to extend these results to a larger population, the current findings are relevant to hip passive devices. The advantages in terms of practicality and usability associated with passive devices with respect to active devices could also strengthen and favor their adoption in real world settings, allowing a larger number of people to walk further, thus improving their independence and quality of life.

Author Contributions: F.A.P., G.M., and E.A. conceived and designed the experiments; E.A. and G.M. performed the experiments; F.A.P. and E.A. analysed the data; A.P. contributed materials; F.A.P., G.M., and A.P. wrote the paper. All authors have read and agreed to the published version of the manuscript.

Funding: This work was partially funded by DIH Vicenza and Vicenza City Council (Italy) within the project InnovationLab (DGR 291/2019).

Institutional Review Board Statement: The study was conducted according to the guidelines of the Declaration of Helsinki, and approved by the Institutional Review Board of the Department of Biomedical Sciences of the University of Padova (HEC-DSB/07-181).

Informed Consent Statement: Informed consent was obtained from all subjects involved in the study.

Data Availability Statement: All the data are presented in the manuscript.

Acknowledgments: The authors desire to thank all the subjects that volunteered for the study and Luqman Aziz for reviewing the English style. The authors would also thank Laura Di Liddo for helping with Exoband manufacturing.

Conflicts of Interest: A patent has been filed with the U.S. Patent Office describing the Exoband components documented in this manuscript, F.A.P. was authors of this patent. F.A.P. and E.A. are employed by Moveo, the company that manufactures the Exoband. There is no other competing interest to declare.

\section{References}

1. Studenski, S.; Perera, S.; Patel, K.; Rosano, C.; Faulkner, K.; Inzitari, M.; Brach, J.; Chandler, J.; Cawthon, P.; Connor, E.B.; et al. Gait speed and survival in older adults. JAMA J. Am. Med. Assoc. 2011, 305, 50-58. [CrossRef] [PubMed]

2. Cesari, M.; Kritchevsky, S.B.; Penninx, B.W.H.J.; Nicklas, B.J;; Simonsick, E.M.; Newman, A.B.; Tylavsky, F.A.; Brach, J.S.; Satterfield, S.; Bauer, D.C.; et al. Prognostic value of usual gait speed in well-functioning older people-Results from the health, aging and body composition study. J. Am. Geriatr. Soc. 2005, 53, 1675-1680. [CrossRef] [PubMed]

3. Brach, J.S.; VanSwearingen, J.M. Interventions to Improve Walking in Older Adults. Curr. Transl. Geriatr. Exp. Gerontol. Rep. 2013, 2, 230-238. [CrossRef] [PubMed]

4. Franceschini, M.; Rampello, A.; Agosti, M.; Massucci, M.; Bovolenta, F.; Sale, P. Walking Performance: Correlation between Energy Cost of Walking and Walking Participation. New Statistical Approach Concerning Outcome Measurement. PLoS ONE 2013, 8. [CrossRef] [PubMed]

5. Schrack, J.A.; Simonsick, E.M.; Ferrucci, L. The energetic pathway to mobility loss: An emerging new framework for longitudinal studies on aging. J. Am. Geriatr. Soc. 2010, 58, S329. [CrossRef]

6. Wert, D.M.; Brach, J.S.; Perera, S.; VanSwearingen, J. The association between energy cost of walking and physical function in older adults. Arch. Gerontol. Geriatr. 2013, 57, 198-203. [CrossRef]

7. Fritz, S.; Lusardi, M. White paper: "walking speed: The sixth vital sign". J. Geriatr. Phys. Ther. 2009, 32, 2-5. [CrossRef]

8. Giovacchini, F.; Vannetti, F.; Fantozzi, M.; Cempini, M.; Cortese, M.; Parri, A.; Yan, T.; Lefeber, D.; Vitiello, N. A light-weight active orthosis for hip movement assistance. In Proceedings of the Robotics and Autonomous Systems; Elsevier: Amsterdam, The Netherlands, 2015; Volume 73, pp. 123-134.

9. Mooney, L.M.; Rouse, E.J.; Herr, H.M. Autonomous exoskeleton reduces metabolic cost of human walking. J. Neuroeng. Rehabil. 2014, 11. [CrossRef]

10. Panizzolo, F.A.; Galiana, I.; Asbeck, A.T.; Siviy, C.; Schmidt, K.; Holt, K.G.; Walsh, C.J. A biologically-inspired multi-joint soft exosuit that can reduce the energy cost of loaded walking. J. Neuroeng. Rehabil. 2016, 13. [CrossRef]

11. Collins, S.H.; Bruce Wiggin, M.; Sawicki, G.S. Reducing the energy cost of human walking using an unpowered exoskeleton. Nature 2015, 522, 212-215. [CrossRef]

12. Seo, K.; Lee, J.; Lee, Y.; Ha, T.; Shim, Y. Fully autonomous hip exoskeleton saves metabolic cost of walking. In Proceedings of the IEEE International Conference on Robotics and Automation, Stockholm, Sweden, 16-21 May 2016; pp. 4628-4635. 
13. Etenzi, E.; Borzuola, R.; Grabowski, A.M. Passive-elastic knee-ankle exoskeleton reduces the metabolic cost of walking. J. Neuroeng. Rehabil. 2020, 17. [CrossRef]

14. Nasiri, R.; Ahmadi, A.; Ahmadabadi, M.N. Reducing the energy cost of human running using an unpowered exoskeleton. IEEE Trans. Neural Syst. Rehabil. Eng. 2018, 26, 2026-2032. [CrossRef]

15. Panizzolo, F.A.; Bolgiani, C.; Di Liddo, L.; Annese, E.; Marcolin, G. Reducing the energy cost of walking in older adults using a passive hip flexion device. J. Neuroeng. Rehabil. 2019, 16, 1-9. [CrossRef]

16. Panizzolo, F.A.; Cimino, S.; Pettenello, E.; Belfiore, A.; Petrone, N.; Marcolin, G. Effect of a passive hip exoskeleton on walking distance in neurological patients. Assist. Technol. 2021. [CrossRef]

17. Sawicki, G.S.; Beck, O.N.; Kang, I.; Young, A.J. The exoskeleton expansion: Improving walking and running economy. J. Neuroeng. Rehabil. 2020, 17, 25. [CrossRef] [PubMed]

18. Panizzolo, F.A.; Freisinger, G.M.; Karavas, N.; Eckert-Erdheim, A.M.; Siviy, C.; Long, A.; Zifchock, R.A.; LaFiandra, M.E.; Walsh, C.J. Metabolic cost adaptations during training with a soft exosuit assisting the hip joint. Sci. Rep. 2019, 9. [CrossRef]

19. Zhang, J.; Fiers, P.; Witte, K.A.; Jackson, R.W.; Poggensee, K.L.; Atkeson, C.G.; Collins, S.H. Human-in-the-loop optimization of exoskeleton assistance during walking. Science 2017, 356, 1280-1283. [CrossRef] [PubMed]

20. Ding, Y.; Kim, M.; Kuindersma, S.; Walsh, C.J. Human-in-the-loop optimization of hip assistance with a soft exosuit during walking. Sci. Robot. 2018, 3. [CrossRef]

21. Young, A.J.; Ferris, D.P. State of the art and future directions for lower limb robotic exoskeletons. IEEE Trans. Neural Syst. Rehabil. Eng. 2017, 25, 171-182. [CrossRef] [PubMed]

22. Dembia, C.L.; Silder, A.; Uchida, T.K.; Hicks, J.L.; Delp, S.L. Simulating ideal assistive devices to reduce the metabolic cost of walking with heavy loads. PLoS ONE 2017, 12. [CrossRef]

23. Himann, J.E.; Cunningham, D.A.; Rechnitzer, P.A.; Paterson, D.H. Age-related changes in speed of walking. Med. Sci. Sports Exerc. 1988, 20, 161-166. [CrossRef]

24. Oberg, T.; Karsznia, A.; Oberg, K. Basic Gait Parameters: Reference Data for Normal Subjects, 10-79 Years of Age. J. Rehabil. Res. 1993, 30 .

25. Brockway, J.M. Derivation of formulae used to calculate energy expenditure in man. Hum. Nutr. Clin. Nutr. 1987, 41, 463-471. [PubMed]

26. Faul, F.; Erdfelder, E.; Lang, A.-G.; Buchner, A. G*Power 3: A flexible statistical power analysis program for the social, behavioral, and biomedical sciences. Behav. Res. Methods 2007, 39, 175-191. [CrossRef] [PubMed]

27. Hopkins, W.G.; Marshall, S.W.; Batterham, A.M.; Hanin, J. Progressive statistics for studies in sports medicine and exercise science. Med. Sci. Sports Exerc. 2009, 41, 3-12. [CrossRef]

28. Barazesh, H.; Sharbafi, M.A. A biarticular passive exosuit to support balance control can reduce metabolic cost of walking. Bioinspir. Biomim. 2020, 15. [CrossRef] [PubMed]

29. Xiong, C.; Zhou, T.; Zhou, L.; Wei, T.; Chen, W. Multi-articular passive exoskeleton for reducing the metabolic cost during human walking. In Proceedings of the 2019 Wearable Robotics Association Conference, WearRAcon 2019, Scottsdale, AZ, USA, 26-28 March 2019; pp. 63-67.

30. Kuo, A.D. A simple model of bipedal walking predicts the preferred speed-step length relationship. J. Biomech. Eng. 2001, 123, 264-269. [CrossRef] [PubMed]

31. Sawicki, G.S.; Ferris, D.P. Mechanics and energetics of level walking with powered ankle exoskeletons. J. Exp. Biol. 2008, 211, 1402-1413. [CrossRef]

32. Koller, J.R.; Jacobs, D.A.; Ferris, D.P.; Remy, C.D. Learning to walk with an adaptive gain proportional myoelectric controller for a robotic ankle exoskeleton. J. Neuroeng. Rehabil. 2015, 12. [CrossRef] [PubMed] 